ThE alveolar macrophage (AM) secretes interleukin $1 \beta$ (IL-1 $\beta$ ), tumour necrosis factor- $\alpha$ (TNF- $\alpha$ ), interleukin-6 (IL-6) and interleukin-8 (IL-8), all of them inflammatory cytokines involved in the pathogenesis of many lung diseases. The aim of the present work was to evaluate the basal and stimulated secretion of these cytokines by human AMs. Human AMs were collected by bronchoalveolar lavage (BAL) from four healthy controls and 13 patients with diffuse interstitial lung disease (five cases of sarcoidosis, three of hypersensitivity pneumonitis and five of idiopathic pulmonary fibrosis). AMs were cultured in the presence or absence of different concentrations of lipopolysaccharide (LPS), phorbolmyristate and gammainterferon. IL-1 $\beta$, TNF- $\alpha$, IL-6 and IL-8 levels were measured in BAL fluid and culture supernatant using specific enzyme-linked immunosorbent assays. The substance found to stimulate the secretion of inflammatory cytokines to the greatest extent was LPS at a concentration of $10 \mu \mathrm{g} / \mathrm{ml}$. Regarding the secretion of IL-1 $\beta$, four observations were of interest: basal secretion was very low; LPS exerted a potent stimulatory effect; considerable within-group variability was observed; and there were no significant differences in the comparisons among groups. With respect to TNF$\alpha$ secretion, the results were similar. The only striking finding was the higher basal secretion of this cytokine with respect to that of $I L-1 \beta$. Regarding the secretion of IL-6, the same pattern followed by TNF- $\alpha$ was found. However, it should be stressed that the increase induced by LPS was smaller than in the two previous cytokines. Regarding the secretion of IL-8, three findings were patent: the strong basal secretion of this cytokine; the moderate increase induced by LPS; and the existence of significant differences among the different groups with respect to the stimulated secretion of this cytokine, which reached maximum values in patients with idiopathic pulmonary fibrosis. Finally, it should be noted that the pattern of cytokines observed in the BAL fluid was similar to that found in cultured AM supernatants. The pattern of inflammatory cytokine secretion by AMs differs from that of other cells of the mononuclear phagocyte system (MPS). In this sense. AMs secrete low amounts of $\mathrm{IL}-1$, moderate amounts of TNF- $\alpha$ and IL-6, and high quantities of IL-8. Adherence is an important stimulus in the secretion of these molecules and LPS elicits an increased secretion inverse to the basal secretion. There is considerable individual variability in the secretion of inflammatory cytokines by the AMs of patients with interstitial lung disease and the AMs of these patients are primed in vivo for the secretion of these cytokines. The results of our study, carried out in vitro, can be extrapolated to the in vivo setting.

Key words: Alveolar macrophage, Tumour necrosis factor, Interleukin-1, Interleukin-6, Interleukin-8

\section{Evaluation of inflammatory cytokine secretion by human alveolar macrophages}

\author{
J. E. Losa García, ${ }^{1, C A}$ F. M. Rodríguez, ${ }^{2}$ \\ M. R. Martín de Cabo, ${ }^{3}$ M. J. García Salgado, ${ }^{4}$ \\ J. P. Losada, ${ }^{4}$ L. G. Villarón, ${ }^{4}$ A. J. López ${ }^{4}$ and \\ J. L. P. Arellano 5
}

${ }^{1}$ Internal Medicine Unit, Fundación Hospital de Alcorcón, Budapest 1, 28922 Alcorcón, Madrid; ${ }^{2}$ Hospital General de Albacete; ${ }^{3}$ Centro de Salud Miguel Servet, Alcorcón; ${ }^{4}$ Department of Medicine, University of Salamanca; ${ }^{5}$ Department of Clinical

Science-1, University of Las Palmas de Gran Canaria, Spain

\footnotetext{
${ }^{\mathrm{CA}}$ Corresponding Author

Tel: (+34) 916219400

Fax: (+34) 916219901

Email: jelosa@fhalcorcon.es
} 


\section{Introduction}

The alveolar macrophage (AM) is the main representative of the mononuclear phagocyte system (MPS) in the lung. ${ }^{1,2}$ Since in healthy subjects more than $90 \%$ of the cells lining the alveolar region are AMs and since the density of this cell line in the fluid covering the alveolar surface is $17 \times 10^{3}$ cells $/ \mu$ l (a concentration 25 -fold higher than that of monocytes in peripheral blood), these MPS cells represent an important sentinel system against inhaled environmental antigens. ${ }^{3,4}$ They play a role in initiating and maintaining an inflammatory response in the lower respiratory tract, ${ }^{5}$ and in global terms they are the most abundant cells in the distal airways in all types of interstitial lung disease (ILD). This cell type synthesizes and secretes all the main types of inflammatory cytokines; that is, IL- $1 \beta$, TNF- $\alpha$, IL-6 and IL-8, ${ }^{5,6}$ well-known mediators of the pulmonary inflammatory response. ${ }^{6,7}$ Diffuse ILDs are a representative example of the pathogenic importance of these cytokines, ${ }^{7}$ above all in the basic initial pathogenic phenomenon in all diffuse ILDs, i.e. alveolitis or inflammation of the distal airways.

The aim of the present work was to evaluate the basal and stimulated secretion of inflammatory cytokines by human AMs from healthy controls and patients with different types of diffuse ILD. In an exhaustive review of the literature we were unable to locate any work addressing simultaneously and exclusively the production of these four cytokines by human AMs in different ILDs.

\section{Methods}

\section{Subjects}

All subjects participating in the study were informed about the nature of the procedure (bronchoscopy and bronchoalveolar lavage) and its complications and written consent to participate was obtained from all of them. None of them smoked or had received pharmacological treatment over the 2 months prior to the study.

\section{Control subjects (Group 1)}

For ethical reasons, the control group only included patients subjected to diagnostic bronchoscopy, in all cases due to chronic cough. The group comprised four subjects in which simple chest X-ray, functional respiratory tests and the bronchoscopic study were normal.

\section{Sarcoidosis patients (Group 2)}

This study population consisted of five patients. Owing to the small number of cases the patients were not further subclassified. Diagnosis was made based on the following criteria: compatible clinical history and chest X-ray; lung or peripheral biopsy demonstrating non-caseating epithelioid granulomas with giant cell formation and a coexisting morphology of the parenchyma compatible with sarcoidosis; no history of mycobacterial, fungal or parasitic infections; no history of exposure to organic or inorganic material known to cause granulomatous lung diseases, and abnormal pulmonary functions with either reduced lung volumes or impairment in diffusion capacity.

\section{Hypersensitivity pneumonitis (HP) patients (Group 3)}

This study population consisted of three patients. Diagnosis was made on the basis of standard parameters: a documented history of exposure to HP antigens; a symptomatic acute episode with chills, fever, cough and breathlessness 4-8 h after exposure to the specific antigen; radiologic and/or functional features of ILD; serum antibodies against antigens, and transbronchial biopsy in agreement with HP.

\section{Idiopathic pulmonary fibrosis (IPF) patients (Group 4)}

This population comprised five patients. Diagnosis of IPF required the following: no clinical history of exposure to environmental agents known to cause interstitial lung disease; no history of chronic lung infections; evidence of interstitial infiltrates at chest X-ray; lung physiology consistent with a restricted ventilatory defect, including decreased lung volume, normal flow rates and/or decreased single-breath carbon monoxide diffusion test; and biopsy evidence in each patient of interstitial pneumonitis with varying degrees of interstitial fibrosis without evidence of granulomas, vasculitis or inorganic material as visualized by polarized light microscopy.

\section{Recovery of alveolar macrophages}

Alveolar macrophages were obtained by fibre optic bronchoscopy with bronchoalveolar lavage (BAL). BAL was performed according to the technical recommendations and guidelines for bronchoalveolar lavage of the Task Group on BAL of the European Society of Pneumology. Premedication was accomplished with intramuscular injection of atropine $30 \mathrm{~min}$ before BAL was performed. During bronchoscopy, topical anaesthesia was administered in several 'bolues' with $2 \%$ lidocaine. Two hundred $\mathrm{ml}$ of sterile physiological saline solution was instilled in four aliquots of $50 \mathrm{ml}$. The site chosen for the lavage was selected attending to the zone with the highest activity detected with other techniques, mainly chest $\mathrm{X}$-ray and gammagraphy with ${ }^{67}$ gallium; in cases with 
diffuse lesions, lavage was performed in the middle lobe or lingula. The fluid was recovered by manual aspiration with a syringe and placed in a sterile siliconized flask at $4^{\circ} \mathrm{C}$.

The fluid thus obtained was filtered through two layers of sterile surgical gauze and then centrifuged at $500 \mathrm{~g}$ over $10 \mathrm{~min}$ at $4^{\circ} \mathrm{C}$. This procedure afforded two fractions: the supernatant and the cellular pellet. The cells were resuspended, depending on the size of the cellular pellet, in 1-5 ml of sterile phosphate buffered solution. The supernatant from the BAL fluid was frozen at $-70^{\circ} \mathrm{C}$ until study. This fluid was later used for the determination of cytokine concentrations in selected cases.

Quantification of the total number of cells was performed using a Neubauer chamber. Cell viability was assessed by trypan blue exclusion and differential cell counts were performed on Diff Quik ${ }^{\circledR}$-stained glass-cover Cytospin slide preparations. In all cases, $\alpha$-naphthyl-esterase (Sigma Chemicals) staining was performed to confirm the suitability of the differential cell count for cells of the mononuclear phagocyte line.

\section{Culture of alveolar macrophages}

Initially, the mononuclear fraction present in the BAL fluid was obtained by separation with a FicollHypaque density gradient. The mononuclear cells were resuspended in culture medium containing, as well as commercial RPMI 1640, glutamine at $2 \mathrm{mM}$, $100 \mathrm{U}$ of penicillin $/ \mathrm{ml}, 100 \mu \mathrm{g}$ of streptomycine $/ \mathrm{ml}$ and $10 \%$ decomplemented fetal calf serum. Following this, 500000 AMs diluted in complete medium were added to each well of the culture dishes up to a total volume of $2 \mathrm{ml}$. This suspension was incubated for $2 \mathrm{~h}$ at $37^{\circ} \mathrm{C}$ in a humid atmosphere with $5 \% \mathrm{CO}_{2}$ for the macrophages to adhere. The supernatant was aspirated out and designated 'preincubation'; this corresponded to cell secretion or elimination in the absence of stimuli. In each case, the following were added to purified macrophages diluted in complete medium: endotoxin (LPS) at final concentrations of $100 \mu \mathrm{g} / \mathrm{ml}, 10 \mu \mathrm{g} / \mathrm{ml}$ and $1 \mu \mathrm{g} / \mathrm{ml}$, phorbolmyristate (PMA) at final concentrations of $10^{-4} \mathrm{M}, 10^{-5} \mathrm{M}$ and $10^{-6} \mathrm{M}$, and human gamma-interferon at final concentrations of $1000 \mathrm{U} / \mathrm{ml}$, and $100 \mathrm{U} / \mathrm{ml}$ and $100 \mathrm{U} / \mathrm{ml}$. The cells were maintained at $37^{\circ} \mathrm{C}$ in a humid atmosphere with $5 \% \mathrm{CO}_{2}$ for $18 \mathrm{~h}$. Supernatants were centrifuged at $500 \mathrm{~g}$ for $10 \mathrm{~min}$ to eliminate aspirated cells and stored at $-70^{\circ} \mathrm{C}$ until later determination. Contamination with endotoxin was ruled out in all cases that were not stimulated with LPS.

\section{Determination of cytokines}

The inflammatory cytokines IL-1 $\beta$, TNF- $\alpha$, IL- 6 and IL8 were determined by direct double-sandwich enzyme-linked immunosorbent assay (ELISA) of antibodies in all the fluids from the BALs, in all the supernatants from the macrophage cultures, and in selected samples of culture medium and 'preincubation' medium from one case in each group (the one with the highest secretion of cytokines in culture). BIOTRAK ${ }^{\circledR}$ (Amersham, UK) equipment was used; this is specific for human IL- $1 \beta$, TNF- $\alpha$, IL-6 and IL-8, with no cross-reactivity among these nor with other cytokines, with a global variation coefficient of less than $10 \%$ and the following respective sensitivity limits: $0.3 \mathrm{pg} / \mathrm{ml} ; 4.8 \mathrm{pg} / \mathrm{ml} ; 0.35 \mathrm{pg} / \mathrm{ml}$ and $4.7 \mathrm{pg} / \mathrm{ml}$. All samples were measured in duplicate. The results were expressed in pico- or nanograms $/ 10^{6} \mathrm{AMs}$ in the case of the culture supernatants and in picograms $/ \mathrm{ml}$ in the case of the BAL fluid. None of the components of the culture medium (RPMI 1640, antibiotics, glutamine or fetal calf serum) displayed immunoreactivity with the four types of cytokine studied in the ELISA assays.

\section{Data statistics}

The data were summarized as means and standard errors of means (SEM) in the different experimental groups. Although all the samples were distributed normally, owing to the low number of cases the Wilcoxon test was used as a non-parametric test for comparison of paired data. However, for the analysis of global differences among groups, ANOVA was used; where global significant differences were found the PLSD Fisher test was employed to determine between which groups the differences existed. In all cases a significance level of $\alpha=0.05$ was considered.

\section{Results}

Table 1 summarizes the basic data (cells recovered and differential counts) about the cases included in the study.

\section{Initial release of cytokines in the absence of stimuli}

In order to determine whether AMs release inflammatory cytokines in the absence of exogenous stimuli or after prolonged culture, cytokines were measured in the supernatant of mononuclear cells incubated for $2 \mathrm{~h}$ for macrophage adherence to occur. Although the aim of this determination was to assess spontaneous release of cytokines by $\mathrm{AM}$, it should be noted that under these conditions cytokines produced by lymphocytes and/or the actual adherence of AMs to the polystyrene may act as modulatory factors.

The results obtained in the index cases of each group afforded interesting data. First, none of the 
Table 1. Basic bronchoalveolar data of the cases studied

\begin{tabular}{|c|c|c|c|c|c|c|c|c|c|c|}
\hline & \multicolumn{2}{|c|}{$\begin{array}{l}\text { Cells } \times \\
10^{6} / \mathrm{ml}\end{array}$} & \multicolumn{2}{|c|}{$\begin{array}{c}\text { Macrophages } \\
(\%)\end{array}$} & \multicolumn{2}{|c|}{$\begin{array}{l}\text { Lymphocytes } \\
(\%)\end{array}$} & \multicolumn{2}{|c|}{$\begin{array}{l}\text { Neutrophils } \\
(\%)\end{array}$} & \multicolumn{2}{|c|}{$\begin{array}{c}\text { Eosinophils } \\
(\%)\end{array}$} \\
\hline & Mean & SEM & Mean & SEM & Mean & SEM & Mean & SEM & Mean & SEM \\
\hline Control $(n=4)$ & 0.24 & 0.05 & 95.7 & 0.7 & 3.5 & 0.6 & 0.7 & 0.5 & 0 & 0 \\
\hline Sarcoidosis $(n=5)$ & 0.38 & 0.07 & 70.6 & 5.6 & 26.6 & 5.6 & 1.4 & 0.5 & 1.2 & 0.9 \\
\hline $\mathrm{HP}(n=3)$ & 0.71 & 0.22 & 35.3 & 11.6 & 53.6 & 0.9 & 9.0 & 7.5 & 2.0 & 0.6 \\
\hline $\operatorname{IPF}(n=5)$ & 0.40 & 0.21 & 84.0 & 5.9 & 3.6 & 1.4 & 9.0 & 2.3 & 3.4 & 0.7 \\
\hline
\end{tabular}

Abbreviations: HP = hypersensitivity pneumonitis; IPF = idiopathic pulmonary fibrosis; SEM = standard error of mean.

subjects studied showed initial spontaneous release of interleukin $1 \beta$. The spontaneous release of TNF- $\alpha$ ranged between 129 and $996 \mathrm{pg} / 10^{6} \mathrm{AMs}$, being maximum in the group of patients with idiopathic pulmonary fibrosis. The relationship between basal secretion after prolonged culture $(18 \mathrm{~h})$ and initial spontaneous release was on average $4: 1$, this ratio being lower in the group of patients with idiopathic pulmonary fibrosis (ratio, 2.3:1). In the case of IL-6, initial spontaneous release ranged between 20 and $421 \mathrm{pg} / 10^{6} \mathrm{AMs}$, being maximum in the group with idiopathic pulmonary fibrosis. The ratio between basal secretion in culture and initial spontaneous release was on average $35: 1$, this ratio decreasing considerably in the patients with hypersensitivity pneumonitis (ratio, 4:1) and being lower in the patients with sarcoidosis (ratio, 16:1).

Finally, the initial spontaneous release of IL-8 ranged between 1861 and $6390 \mathrm{pg} / 10^{6} \mathrm{AMs}$, being maximum in the group with idiopathic pulmonary fibrosis. The relationship between basal secretion in culture and initial spontaneous release was on average 125-fold, this being lower in the group of hypersensitivity pneumonitis patients (ratio, 64:1).

Determination of the type of stimulus and most effective concentration on the secretion of inflammatory cytokines

Three classic stimuli (lipopolysaccharide, phorbolmyristate acetate and gamma-interferon) were evaluated at three different concentrations. Overall, the results obtained (not shown) indicated that the most important stimulus of cytokine secretion was LPS. Regarding the most stimulatory concentration, the overall data indicated that, for this stimulus, it was $10 \mu \mathrm{g} / \mathrm{ml}$. Considering all four cytokines globally, the most stimulatory concentrations of phorbolmyristate acetate and gamma-interferon were respectively $10^{-6} \mathrm{M}$ and $100 \mathrm{U} / \mathrm{ml}$. Figure 1 shows the results of a typical case.

\section{Secretion of inflammatory cytokines under} conditions of stimulation

In Figs 2-5 are represented the results of the basal and stimulated macrophage secretion of the four inflammatory cytokines studied.

Overall comparison of the secretion of the different cytokines revealed that under basal conditions AMs secrete low amounts of IL- $1 \beta$ (in the $10-200 \mathrm{pg} / 10^{6}$ cells range), higher amounts of TNF- $\alpha$ and IL- 6 (in the $1-10 \mathrm{ng} / 10^{6}$ cells range) and large amounts of IL-8 (in the $150-1000 \mathrm{ng} / 10^{6}$ cells range).

Statistical study of the effect of the different stimuli on the secretion of all the cytokines studied disclosed statistically significant differences (Wilcoxon test) between basal secretion and secretion stimulated by LPS or phorbolmyristate $(P<0.008$ in all cases $)$. However, no significant differences between basal secretion and secretion stimulated by gamma-interferon were observed.

On comparing the secretion induced by LPS and phorbolmyristate, statistically significant differences (Wilcoxon test $P<0.004$ in all cases) were observed

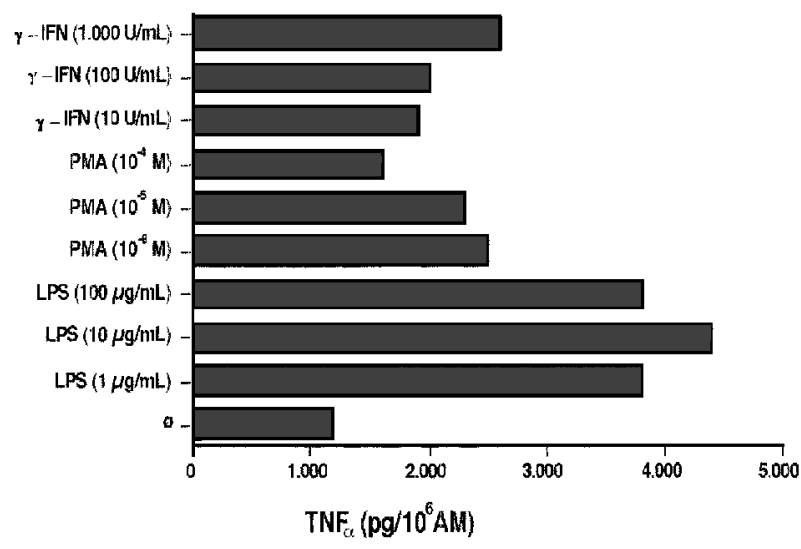

FIG. 1. Effect of concentration and type of stimulus on cytokine secretion by alveolar macrophages (representative case). The most potent stimulus of TNF- $\alpha$ secretion by AM was LPS at a concentration of $10 \mu \mathrm{g} / \mathrm{ml}$. Definition of abbreviations: $\mathrm{AM}=$ alveolar macrophage, IFN = interferon, LPS = lypopolysaccharide, PMA = phorbolmyristate. 


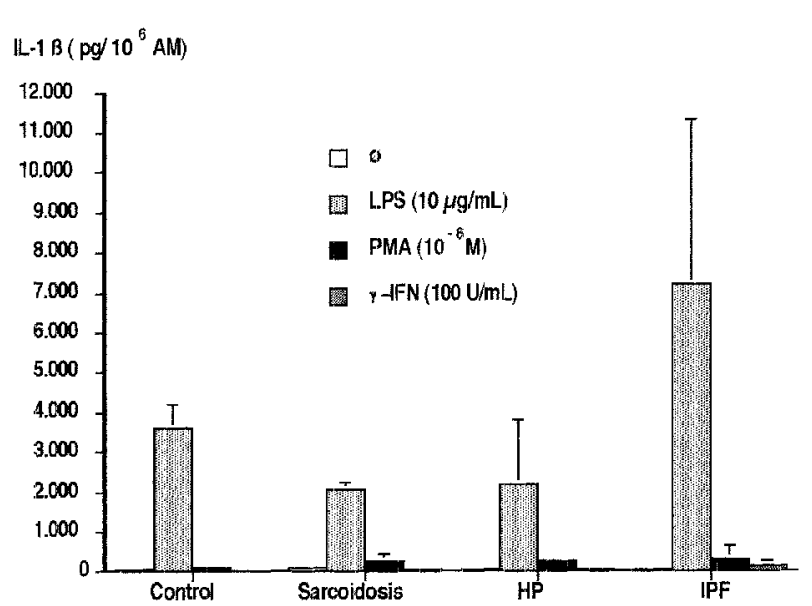

FIG. 2. Interleukin-1 $\beta$ secretion in the different experimental groups. Statistical study of the effect of the different stimuli on IL-1 $\beta$ secretion by AMs revealed significant differences (Wilcoxon test) between basal secretion and that stimulated by LPS $(P<0.001)$ or by phorbolmyristate $(P=0.001)$. No significant differences were observed between basal secretion and that elicited by interferon. On comparing LPSinduced secretion with that induced by PMA, the existence of statistically significant differences was observed (Wilcoxon test, $P=0.001)$. Comparison among the different groups did not reveal significant differences under either basal or stimulated conditions. Definition of abbreviations: $\mathrm{AM}=$ alveolar macrophage, $\mathrm{HP}=$ hypersensitivity pneumonitis, IPF = idiopathic pulmonary fibrosis, IFN = interferon, IL = interleukin, LPS = lipopolysaccharide, PMA, phorbolmyristate.

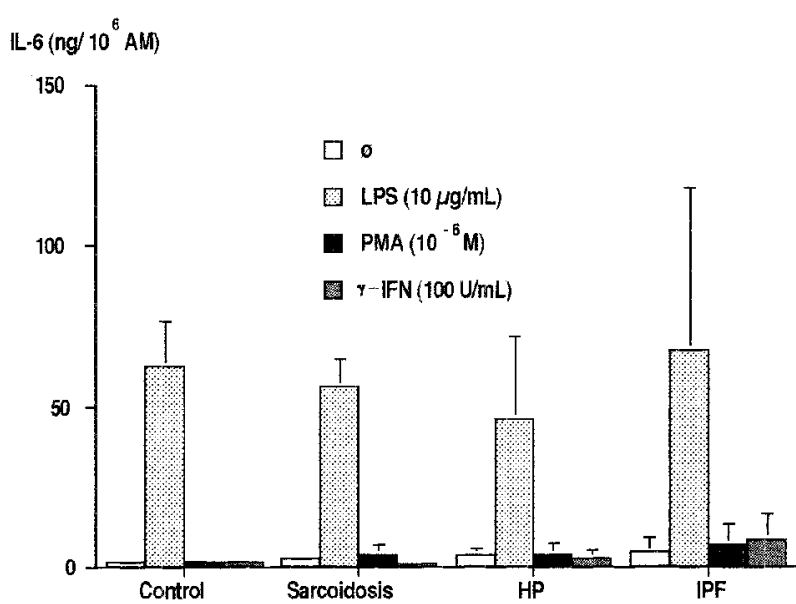

FIG. 4. Interleukin-6 secretion in the different experimental groups. Study of the effect of different stimuli on IL-6 secretion by AMs revealed statistically significant differences (Wilcoxon test, $P=0.001$ for LPS and $P=0.008$ for PMA). No significant differences were found between basal and interferon-induced secretion. Comparison of LPS-induced and PMA-induced secretion revealed statistically significant differences (Wilcoxon test, $P=0.003$ ). Comparison among the different groups study did not disclose significant differences under either basal or stimulated conditions. Definition of abbreviations: $\mathrm{AM}=$ alveolar macrophage, $\mathrm{HP}=$ hypersensitivity pneumonitis, IPF = idiopathic pulmonary fibrosis, IFN = interferon, IL = interleukin, LPS = lipopolysaccharide, PMA, phorbolmyristate.

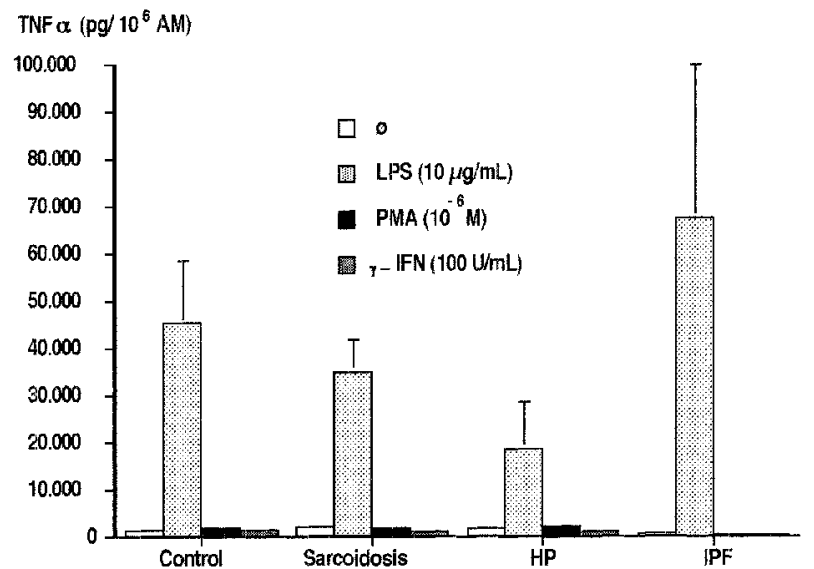

FIG. 3. Tumour necrosis factor- $\alpha$ secretion in the different experimental groups. Study of the effect of different stimuli on TNF- $\alpha$ secretion by AMs revealed statistically significant differences (Wilcoxon test, $P<0.001$ for LPS and $P=0.002$ for PMA). No significant differences were found between basal and interferon-induced secretion. Comparison of LPSinduced and PMA-induced secretion revealed statistically significant differences (Wilcoxon test, $P=0.001$ ). Comparison among the different groups study did not disclose significant differences under either basal or stimulated conditions. Definition of abbreviations: $\mathrm{AM}=$ alveolar macrophage, $\mathrm{HP}=$ hypersensitivity pneumonitis, IPF = idiopathic pulmonary fibrosis, IFN = interferon, LPS = lipopolysaccharide, PMA, phorbolmyristate, TNF = tumour necrosis factor.

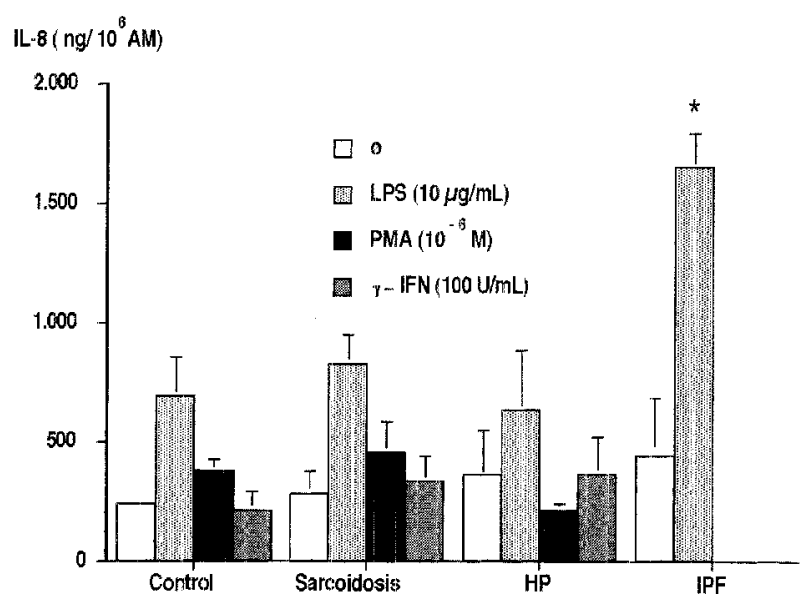

FIG. 5. Interleukin-8 secretion in the different experimental groups. Statistical study of the effect of the different stimuli on IL-8 secretion by AMs revealed significant differences (Wilcoxon test) between basal and LPS-stimulated secretion $(P<0.001)$ or secretion stimulated by PMA $(P=0.004)$. No significant differences between basal and interferon-induced secretion were observed. On comparing LPS-induced secretion and that induced by PMA, statistically significant differences were found (Wilcoxon test, $P=0.004$ ). Comparison of the different groups revealed significant differences in LPS-stimulated secretion $(P=0.025)$. These differences were observed between the patients with IPF and the other groups (Fisher PLSD test $P<0.05$ ). Definition of abbreviations: $\mathrm{AM}=$ alveolar macrophage, $\mathrm{HP}=$ hypersensitivity pneumonitis, IPF = idiopathic pulmonary fibrosis, IFN = interferon, IL = interleukin, LPS = lipopolysaccharide, PMA, phorbolmyristate. 
for all the cytokines studied. Accordingly, with respect to cytokine-stimulated secretion, in all cases the most potent stimulus was found to be LPS, thus confirming preliminary results.

Comparison of the different groups studied as regards inflammatory cytokine secretion did not reveal significant differences in any of them, under either basal or stimulated conditions, with the exception of IL-8 secretion stimulated by LPS. Regarding this, the global differences (ANOVA $P=0.025$ ) were due to the differences between the patients with idiopathic pulmonary fibrosis and the other groups (Fisher PLSD test $P<0.05$ ).

Finally, we evaluated the mean increase in secretion induced by the chemical stimuli. In the case of LPS, this was seen to elicit an approximately 60-fold mean increase in the release of IL-1 $\beta$; a 30-fold increase in that of TNF- $\alpha$; a 20 -fold increase in that of IL- 6 and a three-fold increase in the release of IL-8, all with respect to basal values. However, this mean increase differed depending on the group studied and the type of cytokine in question. Thus, in the case of IL-1 $\beta$ release by LPS stimulation the effect was much greater in the control and IPF groups than in the groups of granulomatous diseases. Moreover, regarding the increase in TNF- $\alpha$ and IL- 8 secretion by LPS the effect was much stronger in the IPF group than in the other groups. With respect to the increase in IL-6 secretion by LPS the effect was much greater in the control than in the other groups. Finally, phorbolmyristate only elicited significant increases in the secretion of IL-1 $\beta$ (tripling its release).

\section{Measurement of cytokines in brochoalveolar lavage fluid}

Cytokine secretion in culture is a widely used method for evaluating AMs in functional terms. However, the complex interplay of interactions among the inflammatory cytokines (inducer or suppressor effects of one cytokine against another) make it necessary to evaluate the net effect of these cytokines in vivo. Accordingly, the concentrations of the four inflammatory cytokines were measured in the BAL fluid. Table 2 shows the mean values. The results were expressed in $\mathrm{pg} / \mathrm{ml}$ of fluid recovered since normalization of the results using albumin as denominator has no biological meaning because the cytokines studied here are not present in the circulating blood of the patients included in the different groups.

Regarding the measurement of IL- $1 \beta$ in the BAL fluid, this cytokine was only detected in one of the four control cases $(1.26 \mathrm{pg} / \mathrm{ml})$; in none of the cases of sarcoidosis; in two out of the three cases of hypersensitivity pneumonitis (1.90 and $3.82 \mathrm{pg} / \mathrm{ml})$ and in two of the five cases of idiopathic pulmonary fibrosis $(0.87$ and $3.95 \mathrm{pg} / \mathrm{ml})$.

TNF- $\alpha$ was detected in the BAL fluid in one of the four control cases $(0.20 \mathrm{pg} / \mathrm{ml})$; in all the cases of sarcoidosis (mean: $2.6 \mathrm{pg} / \mathrm{ml}$; SEM: 0.72 ); in two of the three cases of hypersensitivity pneumonitis $(1.40$ and $3.80 \mathrm{pg} / \mathrm{ml}$ ) and in three of the five cases of idiopathic pulmonary fibrosis $(0.60,2.20$ and $2.21 \mathrm{pg} /$ $\mathrm{ml})$.

In the BAL fluid, IL- 6 was detected in one out of the four control cases $(0.57 \mathrm{pg} / \mathrm{ml})$; in two of the five cases of sarcoidosis $(2.24$ and $2.25 \mathrm{pg} / \mathrm{ml})$, in all the cases of hypersensitivity pneumonitis (mean: $19.21 \mathrm{pg} / \mathrm{ml}$; SEM: 12.68 ) and in all the cases of idiopathic pulmonary fibrosis (mean: $4.98 \mathrm{pg} / \mathrm{ml}$; SEM: 1.98).

Finally, in most of the BAL fluids studied (15 out of 17) the presence of IL- 8 was detected. This magnitude was distributed normally. The inferential statistical study (ANOVA) did not reveal significant overall between-group differences.

\section{Discussion}

\section{Basic aspects of the experimental study}

Selection of the subjects studied here was based on criteria unanimously accepted in the literature. In particular, the AMs from healthy controls and those from patients with three classic types of lung disease: sarcoidosis, hypersensitivity pneumonitis and idiopathic pulmonary fibrosis. The clinical characteristics together with the data on the BAL of the cases studied were similar to those reported in the literature.

Once the present study had been judged suitable, the next step was to standardize the characteristics of cell culture and the concentrations of the most

Table 2. Bronchoalveolar fluid concentration of inflammatory cytokines $(\mathrm{pg} / \mathrm{ml})$

\begin{tabular}{|c|c|c|c|c|c|c|c|c|}
\hline & \multicolumn{2}{|c|}{ IL-1 $\beta$} & \multicolumn{2}{|c|}{ TNF- $\alpha$} & \multicolumn{2}{|c|}{ IL-6 } & \multicolumn{2}{|c|}{ IL-8 } \\
\hline & Mean & SEM & Mean & SEM & Mean & SEM & Mean & SEM \\
\hline Control $(n=4)$ & 0.3 & 0.3 & 0.1 & 0.1 & 0.1 & 0.1 & 112.6 & 80.1 \\
\hline Sarcoidosis $(n=5)$ & 0 & 0 & 2.6 & 0.7 & 0.9 & 0.5 & 34.2 & 15.7 \\
\hline $\mathrm{HP}(n=3)$ & 1.9 & 1.1 & 1.7 & 1.1 & 19.2 & 12.7 & 279.8 & 129.8 \\
\hline IPF $(n=5)$ & 1 & 0.8 & 1 & 0.5 & 5 & 2 & 252.2 & 106.8 \\
\hline
\end{tabular}

Abbreviations: HP = hypersensitivity pneumonitis; IL = interleukin; IPF = idiopathic pulmonary fibrosis; SEM = standard error of mean; TNF = tumour necrosis factor. Invest 1990; 86: 1556-1564. 
appropriate stimuli for the induction of cytokine secretion. Throughout the study, we chose a culture model that used purified AMs. AM purification required three successive steps. First, the mononuclear fraction (macrophages and lymphocytes) was separated of neutrophils, erythrocytes, cellular detritus and dead cells by a density gradient, already reported in the literature for the separation of AMs. ${ }^{1}$ The next step, to separate AMs from lymphocytes, made used of the capacity of AMs to adhere to polystyrene dishes. Both methods permit direct study of cytokine production by AMs. However, the results obtained should be interpreted with caution because adherence itself is able to stimulate the production of these type of inflammatory mediators. ${ }^{5}$ In our opinion, the other option-study of 'fresh', unseparated cells-had more drawbacks as regards interpretation of the results.

\section{Initial spontaneous release of cytokines}

Taking the foregoing data into account, we observed that during the adherence time the AMs secreted very small amounts of the cytokines in comparison with basal secretion in later culture, indicating that this initial release could be discarded in the overall evaluation of the results. On comparing initial spontaneous release among the different groups, in all the cytokines studied the maximum values were found in the group with idiopathic pulmonary fibrosis, reflecting previous stimulation of the macrophages in vivo. Another aspect that can be interpreted in the same sense was the low relationship between initial spontaneous release of IL- 6 and IL- 8 and their basal secretion during prolonged culture in the patients with granulomatous disease. Finally, the relationship between basal secretion in culture and initial spontaneous release was much higher for IL-8, intermediate for IL-6 and low for TNF- $\alpha$, suggesting that adherence to the plastic support is a very important stimulus in the secretion of IL- 8 and, to a lesser extent, that of IL- 6 . Accordingly, from these data it may be concluded that in interstitial lung disease AMs are primed in vivo for the secretion of inflammatory cytokines and that adherence itself strongly induces the secretion of these molecules. In an exhaustive review of the literature, we have found no reference to initial spontaneous release such that no data are available for comparative purposes.

\section{Type and concentration of stimulus}

After the scant initial release had been confirmed, the next aspect to be evaluated was the type and concentration of the agents that would exert a maximum effect for the secretion of the different inflammatory cytokines. The results obtained indicated that the most potent stimulus was LPS. This is consistent with most reports in the literature. Regarding the concentration of this agent, maximum stimulation of cytokine production was achieved with $10 \mu \mathrm{g} / \mathrm{ml}$, a concentration identical to that used by other authors. ${ }^{8,9}$ Phorbolmyristate (PMA), at all concentrations studied, had a less potent effect than LPS, although it did elicit a larger increase in the secretion of the cytokines with respect to basal conditions. However, at all concentrations studied gamma-interferon was unable to increase the secretion of these molecules. These findings are similar to those reported in the literature showing that PMA stimulates IL$1,{ }^{2}$ and TNF- $\alpha^{10}$ secretion while gamma-interferon either fails to affect secretion $^{11}$ or exerts a costimulant effect on other substances, ${ }^{11}$ although other authors have reported a stimulatory effect. ${ }^{10,12}$ Accordingly, LPS at a final concentration of $10 \mu \mathrm{g} / \mathrm{ml}$ was chosen as the best stimulus in the evaluation of cytokine secretion.

\section{Basal and stimulated secretion of cytokines by} alveolar macrophages

The results obtained can be grouped into several different aspects.

First, with all the cytokines studied, the most homogeneous group was found to be the control group, considerable variability being found in the other groups. The latter phenomenon can be attributed to the fact that we were studying patients in different disease stages with different developmental forms (inactive or active). ${ }^{8}$ This variability in the secretion of cytokines has been documented by other authors $^{13}$ and has been attributed to the heterogeneous secretion capacity of AMs themselves. ${ }^{1,2,13}$ Another factor to be considered is that cytokine secretion by AMs may differ within the lungs themselves. $^{14}$

Second, in all cases basal secretion of all the inflammatory cytokines was demonstrated, although the degree of release was different in each one. Thus AMs secrete small amounts of IL-1 $\beta$, moderate amounts of TNF- $\alpha$ and IL-6 and high amounts of IL-8. This type of cytokine profile has been reported by other authors ${ }^{5}$ and has been interpreted in terms of the notion that in the healthy lung aggressive agents will give rise to inflammatory responses at the expense of large amounts of IL- 6 and TNF- $\alpha$ and only limited amounts of IL-1. ${ }^{15}$ By contrast, agents that elicit a recruitment of monocytes to the lung will induce a strong production of IL-1 and will thus facilitate the synergic action of this cytokine with TNF- $\alpha$ and IL- 6 , the local inflammatory response being enhanced. ${ }^{15}$ Evaluation of the concentration of the different inflammatory cytokines in the BAL fluid afforded the same pattern of cytokine secretion, suggesting that to a certain extent secretion in culture would be similar to that taking place in vivo. 
Thirdly, the data concerning the basal and stimulated secretion of the different cytokines are comparable with those described in the literature as regards ELISA measurement in culture supernatants. In this sense, the spontaneous secretion of cytokines in the control group was similar to that reported by Thomassen et al. ${ }^{16}$ but higher than that reported by Standiford et al. ${ }^{6,17,18}$ Agostini et al. ${ }^{9}$ and Homolka ${ }^{19}$. The cytokine concentration range in the AM supernatant of control subjects stimulated with LPS afforded somewhat discrepant results with respect to the literature. In particular, IL-1 $\beta$ levels of between 0.350 and $50 \mathrm{ng} / 10^{6}$ AMs have been reported ${ }^{8,16,20-26}$ while in this study a mean value of $3.6 \mathrm{ng} / 10^{6} \mathrm{AMs}$ was found. Stimulated secretion of TNF- $\alpha$ in the literature ranges between 5 and $100 \mathrm{ng} / 10^{6} \mathrm{AMs},{ }^{8,9,16,21,23,26,27}$ while in this study a mean value of $45 \mathrm{ng} / 10^{6}$ AMs was found. Regarding the stimulated secretion of IL- 6 , the literature contains few references, pointing to a range of $0.2-87 \mathrm{ng} / 10^{6} \mathrm{AMs},{ }^{8,9,15,16,19,25}$ while in this study a mean value of $63 \mathrm{ng} / 10^{6}$ AMs was found. Finally, stimulated IL-8 release in the control group was $692 \mathrm{ng} / 10^{6} \mathrm{AM}$, higher than that reported in the literature (10-500 ng/10 $\left.{ }^{6} \mathrm{AMs}\right){ }^{17,18,28,29}$

The quantitative data on the production of inflammatory cytokines in interstitial lung diseases are more limited than those available for controls. The results obtained concerning the basal and stimulated secretion of cytokines by AMs from patients with sarcoidosis point to both discrepancies and similarities with those referred to in the literature. Thus, our data are similar to those reported by the team of Müller-Quernheim ${ }^{19,30}$ but differ from those of Steffen $e t$ al. ${ }^{8}$ and Nakamura et $a l .{ }^{26}$ We can only compare the results obtained in the groups of patients with hypersensitivity pneumonitis with respect to IL-8 secretion, ${ }^{31}$ since the only studies carried out on the production of cytokines in this process have used bioassays to measure IL-1 and TNF. $^{32}$ Our results on the quantification of the AMmediated basal and stimulated secretion of IL-8 by AMs of patients with hypersensitivity pneumonitis differ considerably from those reported by Denis. ${ }^{31}$ Finally, in idiopathic pulmonary fibrosis the data in the literature about the evaluation of cytokine secretion by immunoassay are scanty. ${ }^{26,33}$ In this sense, our results differ from those of Nakamura ${ }^{26}$ and in comparison with those reported by Zhang et al. ${ }^{33}$ they are similar with respect to TNF- $\alpha$ but differ with respect to IL-1 $\beta$. In no case have we found any references to IL-6 production by AMs in patients with idiopathic pulmonary fibrosis.

Fourthly, between-group comparison of the secretion of the different types of cytokine did not reveal global significant differences with the exception of stimulated IL-8 release. Detailed analysis of the differences revealed that the group with the greatest production of this cytokine included the patients with idiopathic pulmonary fibrosis. Our data differ from some appearing in the literature pointing to an increase in the release of these inflammatory cytokines in sarcoidosis, ${ }^{8,19,26,34}$ hypersensitivity pneumonitis $^{31,32}$ and idiopathic pulmonary fibrosis ${ }^{13,26,33,35}$ with respect to controls. Several reasons may be invoked to account for the discrepancy between our data and these studies. ${ }^{8}$ Firstly, the number of subjects in each group is low and probably, although diagnosis was correct, there would be differences in the degree of activity or in the developmental stage of the diseases. Secondly, not all the authors referred to above used immunoassay as the determination method, hence limiting comparisons. Additionally, the sensitivity of each immunoassay may differ. Thirdly, different AM culture techniques may have been used. Despite these observations, in another series of works on AM cytokine secretion results similar to our own have been reported. ${ }^{19,26,36}$ The increase in the secretion of IL-8 in the group of patients with idiopathic pulmonary fibrosis-also recently reported by Nakamura et al. ${ }^{26}$-which may be involved to a more important extent in the recruitment of neutrophils to the lung, should also be noted; this finding is characteristic in the BAL fluid of this group of patients. ${ }^{26,37,38}$ Currently, the central role of IL-8 in the pathogenesis of this disease seems unquestionable. ${ }^{26,38}$

Finally, we stress that the increase in secretion induced by stimulation with LPS varies for each cytokine and among the different groups. In this sense, the increase is maximum in IL- $1 \beta$ and TNF- $\alpha$, suggesting that in the case of cytokines being produced intensely in vivo (e.g. IL-8) an additional stimulus would elicit a slight increase in release. Moreover, in controls and patients with idiopathic pulmonary fibrosis the endotoxin is able to increase IL-1 $\beta$ secretion to a considerable degree, this increase being much less pronounced in patients with sarcoidosis and pneumonitis. Presumably, this could be interpreted in terms of the idea that AMs would be stimulated in vivo. The decrease in the secretory response of inflammatory interleukins to stimulation with LPS in AMs that under basal conditions secrete large amounts has been described in patients with pneumonia. $^{14}$ This tolerance to the endotoxin is interpreted as a homeostatic response whose aim would be to limit local inflammatory response and hence lung damage. ${ }^{14}$ Other authors have demonstrated that the AMs of patients with ILD can be primed in vivo to produce large amounts of cytokines (IL-1) when stimulated in vitro. ${ }^{39}$

The following conclusions can be drawn from this work. The pattern of inflammatory cytokine secretion by AMs differs from that of others cells belonging to the MPS. Thus, AMs secrete low amounts of IL-1; moderate amounts of TNF and IL- 6 , and high amounts of IL-8. Adherence is an important stimulus in the 
secretion of these molecules and LPS elicits an increase in the inverse sense to basal secretion. Considerably individual variability exists as regards the secretion of inflammatory cytokines by the AMs of patients with interstitial lung disease and the AMs of these patients are primed in vivo for the secretion of these cytokines. The results of our study, performed in vitro, could be extrapolated to the in vivo setting.

\section{References}

1. Ferro TJ, Kern JA, Elias JA, Kamoun M, Daniele RP, Rossman MD. Alveolar macrophages, blood monocytes, and density-fractionated alveolar macrophages differ in their ability to promote lymphocyte proliferation to mitogen and antigen. Am Rev Respir Dis 1987; 135: 682-687.

2. Elias JA, SchrieberAD, Gustilo K. Differential interleukin 1 elaboration by unfractionated and density fractionated human alveolar macrophages and blood monocytes: relationship to cell maturity. J Immunol 1985; 135: 3198-3204

3. Moore SA, Strieter RM, Rolfe MW, Standiford TJ, Burdick MD, Kunkel SL. Expression and regulation of human alveolar macrophage-derived interleukin-1 receptor antagonist. Am J Respir Cell Mol Biol 1992; 6: 569-575.

4. Martinet Y, Yamauchi K, Crystal RG. Differential expression of the tumor necrosis factor/cachectin gene by blood and lung mononuclear phagocytes. Am Rev Respir Dis 1988; 138: 659-665.

5. Thomassen MJ, Meeker DP, Antal JM, Connors MJ, Wiedemann HP Synthetic surfactant (Exosurf) inhibits endotoxin-stimulated cytokine secretion by human alveolar macrophages. Am J Respir Cell Mol Biol 1992; 7: 257-260.

6. Rolfe MW, Kunkel SL, Rowens B, Standiford TJ, Cragoe EJ, Strieter RM Suppression of human alveolar macrophage-derived cytokines by amiloride. Am J Respir Cell Mol Biol 1992; 6: 576-582.

7. Kelley J. Cytokines of the lung. Am Rev Respir Dis 1990; 141: $765-788$

8. Steffen M, Petersen J, Oldigs $\mathrm{M}$, et al. Increased secretion of tumor necrosis factor-alpha, interleukin- 1 beta, and interleukin- 6 by alveolar macrophages from patients with sarcoidosis. J Allergy Clin Immunol 1993; 91: 939-949.

9. Agostini C, Trentin L, Zambello R, et al. Alveolar macrophages in HIV-1 infection express accessory molecules, activation markers, and release increased biological response modifiers. Chest 1993; 103: (Suppl): 108-111

10. Agostini C, Zambello R, Trentin L, et al. Alveolar macrophages from patients with AIDS and AIDS-related complex constitutively synthesize and release tumor necrosis factor alpha. Am Rev Respir Dis 1991; 144: 195-201.

11. Eden E, Turino GM. Interleukin-1 secretion by human alveolar macrophages stimulated with endotoxin is augmented by recombinant immune (gamma) interferon. Am Rev Respir Dis 1986; 133: 455-460.

12. Lacraz S, Nicod L, Galve-de-Rochemonteix B, Baumberger C, Dayer JM, Welgus HG. Suppression of metalloproteinase biosynthesis in human alveolar macrophages by interleukin-4. J Clin Invest 1992; 90: 382-388.

13. Pantelidis P, Southcott AM, Dubois RM. Alveolar macrophages from patients with fibrosing alveolitis secrete more $\mathrm{TNF}_{\mathrm{a}}$ than patients with sarcoidosis and normal individuals. Thorax 1994; 49: 408P.

14. Dehoux MS, Boutten A, Ostinelli J, et al. Compartmentalized cytokine production within the human lung in unilateral pneumonia. Am J Respir Crit Care Med 1994; 150: 710-716

15. Kotloff RM, Little J, Elias JA. Human alveolar macrophage and blood monocyte interleukin-6 production. Am J Respir Cell Mol Biol 1990; 3: 497-505.

16. Thomassen MJ, Meeker DP, Deodhar SD, Wiedemann HP, Barna BP Activation of human monocytes and alveolar macrophages by a synthetic peptide of C-reactive protein. J Immunother 1993; 13: 1-6.

17. Standiford TJ, Kunkel SL, Rolfe MW, Evanoff HL, Allen RM, Strieter RM. Regulation of human alveolar macrophage- and blood monocyte-derived interleukin-8 by prostaglandin E2 and dexamethasone. Am J Respir Cell Mol Biol 1992; 6: 75-81.
18. Standiford TJ, Kunkel SL, Liebler JM, Burdick MD, Gilbert AR, Strieter RM. Gene expression of macrophage inflammatory protein- $1 \alpha$ from human blood monocytes and alveolar macrophages is inhibited by interleukin- 4 . Am J Respir Cell Mol Biol 1993; 9: 192-198.

19. Homolka J, Müller-Quernheim J. Increased interleukin 6 production by bronchoalveolar lavage cells in patients with active sarcoidosis. Lung 1993; 171: 173-183.

20. Iwamoto GK, Monick MM, Burmeister LF, Hunninghake GW. Interleukin 1 release by human alveolar macrophages and blood monocytes. $A m$ J Physiol 1989; 256: C1012-C1015.

21. Sone S, Yanagawa H, Nishioka Y, et al. Interleukin- 4 as a potent downregulator for human alveolar macrophages capable of producing tumour necrosis factor-alpha and interleukin-1. Eur Respir J 1992; 5: 174-181.

22. Allen JN, Herzyk DJ, Wewers MD. Colchicine has opposite effects on interleukin-1 beta and tumor necrosis factor-alpha production. Am J Pbysiol 1991; 261: L315-L321.

23. Nishioka Y, Sone S, Orino E, Nil A, Ogura T. Down-regulation by interleukin 4 of activation of human alveolar macrophages to the tumoricidal state. Cancer Res 1991; 51: 5526-5631.

24. Twigg HL III, Iwamoto GK, Soliman DM. Role of cytokines in alveolar macrophage accessory cell function in HIV-infected individuals. $J$ Immunol 1992; 149: 1462-1469.

25. Soliman DM, Twigg HL III. Cigarette smoking decreases bioactive interleukin-6 secretion by alveolar macrophages. Am J Physiol 1992; 263: L471-L478.

26. Nakamura H, Fujishima S, Waki Y, et al. Priming of alveolar macrophages for interleukin-8 production in patients with idiopathic pulmonary fibrosis. Am J Respir Crit Care Med 1995; 152: 1579-1586.

27. Hirsch CS, Ellner JJ, Russell DG, Rich EA. Complement receptormediated uptake and tumor necrosis factor-alpha-mediated growth inhibition of Mycobacterium tuberculosis by human alveolar macrophages. I Immunol 1994; 152: 743-753.

28. Sylvester I, Rankin JA, Yoshimura T, Tanaka S, Leonard EJ. Secretion of neutrophil attractant/activation protein by lipopolysaccharide-stimulated lung macrophages determined by both enzyme-linked immunosorbent assay and N-terminal sequence analysis. Am Rev Respir Dis 1990; 141: $683-688$.

29. Rankin JA, Sylvester I, Smith S, Yoshimura T, Leonard EJ. Macrophages cultured in vitro release leukotriene B4 and neutrophil attractant/ activation protein (interleukin 8) sequentially in response to stimulation with lipopolysaccharide and zymosan. J Clin Invest 1990; 86: 1556-1564.

30. Muller-Quernheim J, Pfeifer S, Mannel D, Strausz J, Ferlinz R. Lungrestricted activation of the alveolar macrophage/monocyte system in pulmonary sarcoidosis. Am Rev Respir Dis 1992; 145: 187-192.

31. Denis M. Proinflammatory cytokines in hypersensitivity pneumonitis. Am J Respir Crit Care Med 1995; 151: 164-169.

32. Denis M, Bedar M, Laviolette M, Cormier Y. A study of monokine release and natural killer activity in the bronchoalveolar lavage of subjects with farmer's lung. Am Rev Respir Dis 1993; 149: 934-939.

33. Zhang Y, Lee TC, Guillemin B, Yu MC, Rom WN. Enhanced IL-1 and tumor necrosis factor- $\alpha$ release and messenger RNA expression in macrophages from idiopathic pulmonary fibrosis or after asbestos exposure. $J$ Immunol 1993; 150: 4188-4196.

34. Sibille Y, Naegel GP, Merrill WW, Young KR Jr, Care SB, Reynolds-HY Neutrophil chemotactic activity produced by normal and activated human bronchoalveolar lavage cells. J Lab Clin Med 1987; 110: 624-633

35. Meloni F, Ballabio P, Leo G, Bianchi L, Luisetti M, Gialdroni Grassi G. IL-8 production by alveolar macrophages in idiopathic pulmonary fibrosis. Eur Respir J 1993; Suppl 17:528s.

36. Eden E, Turino GM. Interleukin 1 secretion from human alveolar macrophages in lung disease. J Clin Immunol 1986; 6: 326-333.

37. Lynch JP III, Standiford TJ, Rolfe MW, Kunkel SL, Strieter RM. Neutrophilic alveolitis in idiopathic pulmonary fibrosis. The role of interleukin-8. Am Rev Respir Dis 1992; 145: 1433-1439.

38. Southcott AM, Jones KP, Li D, et al. Interleukin 8. Differential expression in lone fibrosing alveolitis and systemic sclerosis. Am J Respir Crit Care Med 1995; 151: 1604-1612.

39. Janson Rw, Hance KR, King TE. Human alveolar macrophages produce predominantly the $35-\mathrm{kD}$ pro-forms of interleukin-1a and interleukin- $1 \mathrm{~b}$ when stimulated with lipopolysaccharide. Am J Respir Crit Care Med 1995; 151: 1613-1620.

\section{Received 5 December 1998; accepted in revised form 5 January 1999}




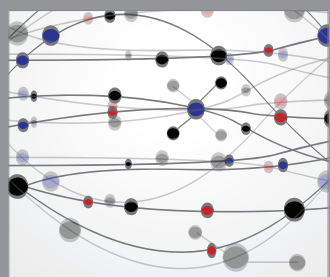

The Scientific World Journal
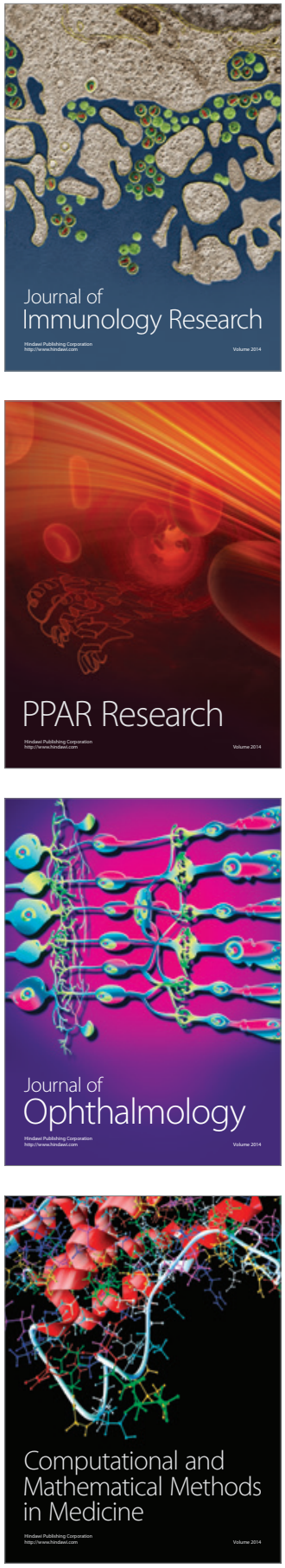

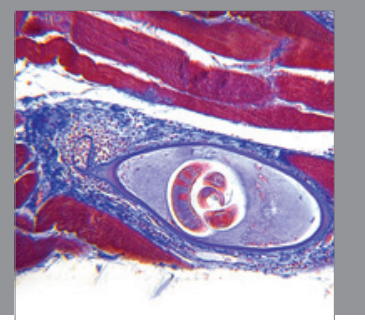

Gastroenterology

Research and Practice
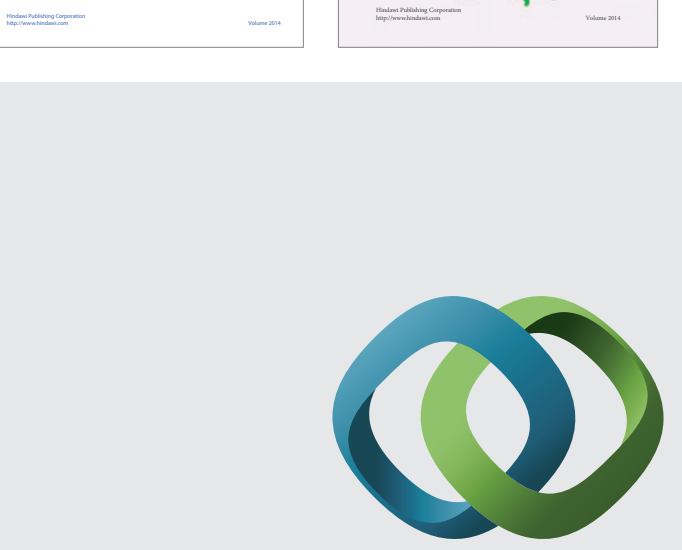

\section{Hindawi}

Submit your manuscripts at

http://www.hindawi.com
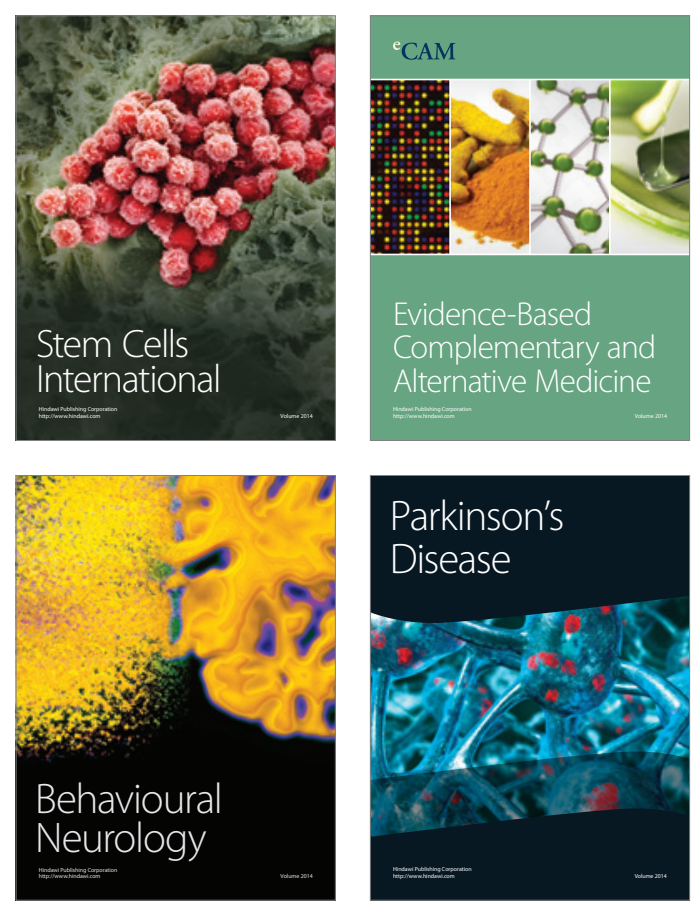

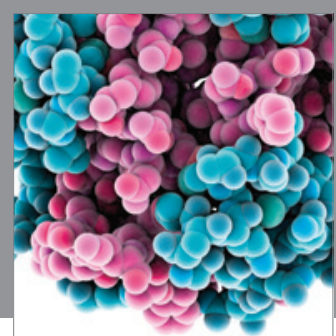

Journal of
Diabetes Research

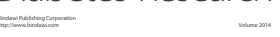

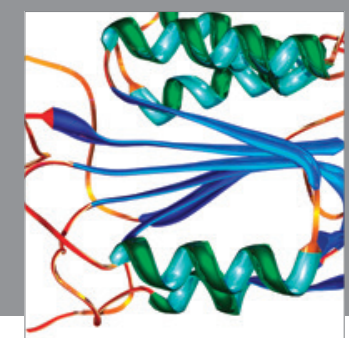

Disease Markers
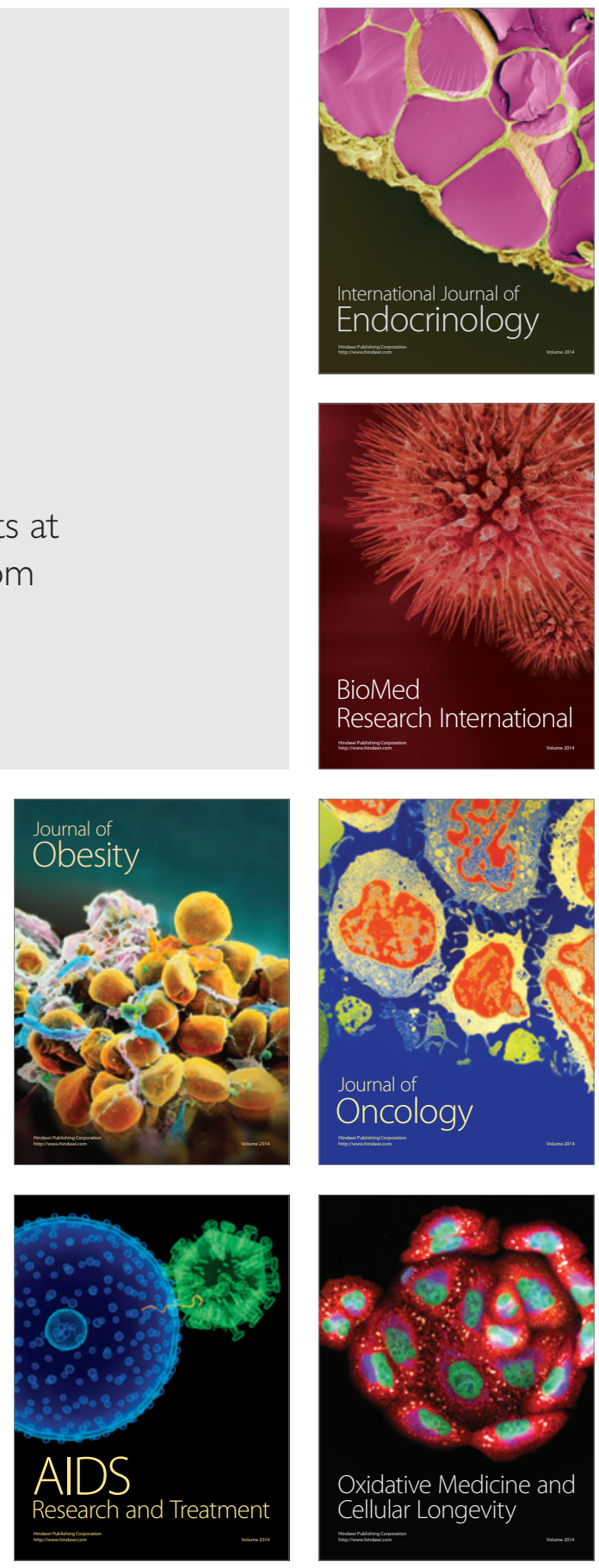